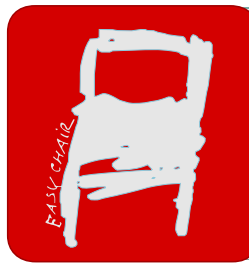

EPiC Series in Health Sciences

Volume 4, 2020, Pages 215-219

CAOS 2020. The 20th Annual Meeting of the International Society for Computer Assisted Orthopaedic Surgery

\title{
Optimizing TKA Positioning via Intraoperative Ligament Characterization
}

\author{
Alexander D. Orsi $\mathrm{PhD}^{1}$, Edgar Wakelin $\mathrm{PhD}^{2}$, Sami Shalhoub $\mathrm{MS}^{2}$, Jeffrey M. \\ Lawrence $\mathrm{MD}^{3}$, Corey E. Ponder $\mathrm{MD}^{4}$, John M. Keggi $\mathrm{MD}^{5}$, and Christopher \\ Plaskos $\mathrm{PhD}^{2}$ \\ 1 Corin Australia, Sydney, New South Wales, Australia \\ alex.orsi@coringroup.com \\ 2 Corin U.S.A, Raynham, Massachusetts, U.S.A. \\ edgar.wakelin@coringroup.com \\ sami.shalhoub@coringroup.com \\ christopher.plaskos@coringroup.com \\ 3 Gundersen Health System, Viroqua, WI, U.S.A \\ 4 Department of Orthopedic Surgery and Rehabilitation, University of Oklahoma Health Sciences \\ Center, Oklahoma City, OK, U.S.A \\ 5 Orthopaedics New England, Middlebury, CT, U.S.A
}

\begin{abstract}
The goal of total knee arthroplasty (TKA) is to position the prosthesis with a balanced soft tissue envelope throughout the flexion cycle. Determining a desirable amount of ligament tension is crucial as instability is a leading cause for revision surgery. This is challenging due to the subject specific, and non-linear, nature of ligament mechanical properties. This study aims to characterize ligament stiffness profiles intraoperatively and identify the stiffness transition point (STP) using a ligament balancing robot. The study will also identify how the surgeon selected joint force relates to the stiffness transition point.

45 patients were reviewed. After the proximal tibial resection, intraoperative assessment of the medial and lateral ligaments was performed via a load cycle ramped up from $50 \mathrm{~N}$ to $120 N$, then down from $120 N$ to $50 N$. This was performed at extension $\left(10^{\circ}\right)$ and in flexion $\left(90^{\circ}\right)$. Force and displacement data were processed to determine the stiffness profiles for the medial and lateral soft tissue envelope. A bilinear fit model was used to determine the slopes and STP.

The average STP was between $83 N$ and $90 N$, varying widely with standard deviations approaching $14 N$. The median joint tension selected by the surgeon was $80 N$. On average the selected joint force was $3 N$ to $8.9 N$ below the STP.

The medial compartment behaved similarly in extension and flexion. The lateral compartment had higher stiffness in extension than in flexion. Across all loading conditions, the down-cycle data was more consistent than the up-cycle data.

The STP is a proposed target for ligament tension as this theoretically avoids the high stiffness regime. The results show the joint tension selected by the surgeons, based on their experience and intraoperative feel, is similar to the STP. Due to the high patient variability
\end{abstract}


observed in the STP, using a patient specific method to determine the joint tension is recommended. Future work will investigate how joint tension relative to the STP affects patient outcomes. This will provide insight into optimizing joint tension during TKA.

\section{Introduction}

Determining a desirable amount of ligament tension is crucial for total knee arthroplasty (TKA) as instability is a leading cause for revision surgery [1,2,3]. Little data exists on optimizing joint space tension during TKA. Traditionally surgeons use manual devices to assess joint space tension. A downside to this is that no prescribed force is targeted. This requires the surgeon to operate based on what force "feels" adequate for the joint space [4].

Ligament behaves as a piece-wise, non-linear visco-hyperelastic material. During axial loading it initially behaves as a non-linear material (toe region) as the collagen fiber network uncrimps. With increased load, the collagen fibers align and the ligament transitions to a linear material [5]. The point at which this behavior change occurs is defined as the stiffness transition point (STP). Ligament properties are subject specific, varying widely based on factors such as sex, BMI and age $[3,6]$. This study aims to characterize ligament stiffness profiles intraoperatively to identify the STP. The study will also compare how the surgeon selected joint force relates to the STP.

\section{Methods}

45 patients were assessed by 2 surgeons at 2 centers. After the proximal tibial resection, and prior to femoral resection, an intraoperative assessment of the medial and lateral ligaments was performed using a digital joint tensioning device (BalanceBot ${ }^{\mathrm{TM}}$ ), seen in Figure 1a. A load cycle was applied independently to both the medial and lateral compartments, ramped up from $50 \mathrm{~N}$ to $120 \mathrm{~N}$, then down from $120 \mathrm{~N}$ to $50 \mathrm{~N}$. This was performed at extension $\left(10^{\circ}\right)$ and in flexion $\left(90^{\circ}\right) \cdot 10^{\circ}$ was chosen in extension to avoid posterior capsule tightening and to isolate the medial and lateral structures. The surgeon then selected a force value to apply to the joint space based on intraoperative feel, and finished the procedure. Force and displacement data was processed to determine the stiffness profiles for the medial and lateral soft tissue envelope in both flexion and extension. A bilinear fit model was used to determine the slopes and stiffness transition points (STP). While it is known that the initial behavior of ligament is non-linear, this fitting process was used to keep consistency with previous work performed by Hesterbeek et al [2]. The average differentials between the STP and the surgeon selected joint force were also calculated.

\section{Results}

Slope 1 refers to the initial low stiffness region, while Slope 2 refers to the high stiffness region. Figure $1 \mathrm{~b}$ provides stiffness profiles from an example patient. The STP is the force value at the intersection point of the two slopes. The average STP ranged between $83 N$ and $90 N$ across all loading conditions, seen in Table 1. High variability was observed for the STP with standard deviations as high as $14 N$. Across all testing conditions, the STP showed greater consistency during down cycle loading compared to up cycle loading.

The median surgeon selected joint tension was $80 \mathrm{~N}$. 24 patients were implanted with $80 \mathrm{~N}$, 18 patients were implanted with $90 N$, and one patient was implanted with $100 N$ of joint tension. 


\begin{tabular}{|c|c|c|c|c|c|c|c|}
\hline \multicolumn{3}{|c|}{ Load Condition } & Slope 1 & Slope 2 & $R^{2}$ & STP & Differential \\
\hline \multirow{4}{*}{ Extension } & \multirow[b]{2}{*}{ Medial } & $\mathrm{Up}$ & $22.7(11.9)$ & $30.7(14.7)$ & 0.9690 & $89.8(13.1)$ & $-8.9(22.7)$ \\
\hline & & Down & $26.7(11)$ & $55.7(27.8)$ & 0.9791 & $87.6(10.9)$ & $-6.7(20.7)$ \\
\hline & \multirow{2}{*}{ Lateral } & $\overline{\mathrm{Up}}$ & $18.3(23.9)$ & $30.3(15.6)$ & 0.9845 & $86.6(13.5)$ & $-5.8(22.2)$ \\
\hline & & Down & $26.5(12.4)$ & $61.8(31.2)$ & 0.9824 & $85.2(11)$ & $-4.3(22)$ \\
\hline \multirow{4}{*}{ Flexion } & \multirow{2}{*}{ Medial } & $\mathrm{Up}$ & $24.2(11.7)$ & $29.2(9)$ & 0.9894 & $87.6(11)$ & $-6.7(22.1)$ \\
\hline & & Down & $23.3(6.9)$ & $50.5(11.9)$ & 0.9661 & $83.9(7.9)$ & $-3.0(16.6)$ \\
\hline & \multirow{2}{*}{ Lateral } & Up & $20.2(21)$ & $17.3(8.2)$ & 0.9870 & $88.6(14)$ & $-7.7(23.5)$ \\
\hline & & Down & $15.8(6.9)$ & 40.7(20.6) & 0.9613 & $83.8(10)$ & $-3.0(22.1)$ \\
\hline
\end{tabular}

Table 1: Stiffness results

Joint tension data was not recorded in two patients. The differential data shows on average the selected joint force was $3 N$ to $8.9 N$ below the STP in all loading conditions.

In extension, the average medial compartment stiffness (up-cycle/down-cycle) of Slope 1 was $23 / 27 \mathrm{~N} / \mathrm{mm}$ with a Slope 2 of $31 / 56 \mathrm{~N} / \mathrm{mm}$. In flexion, the medial compartment stiffness of Slope 1 was $24 / 23 \mathrm{~N} / \mathrm{mm}$ with a Slope 2 of $29 / 51 \mathrm{~N} / \mathrm{mm}$.

In extension, the average lateral compartment stiffness of Slope 1 was $18 / 27 \mathrm{~N} / \mathrm{mm}$ with a Slope 2 of $30 / 62 \mathrm{~N} / \mathrm{mm}$. In flexion, the average lateral compartment stiffness of Slope 1 was $20 / 16 \mathrm{~N} / \mathrm{mm}$ with a Slope 2 of $17 / 41 \mathrm{~N} / \mathrm{mm}$.

Slope 1 was most consistent in flexion on the down cycle with standard deviations of $6.9 \mathrm{~N}$ and $6.9 \mathrm{~N}$ for the medial and lateral ligament respectively. Slope 2 was most consistent in flexion on the up cycle with standard deviations of $9 \mathrm{~N}$ and $8.2 \mathrm{~N}$ for the medial and lateral ligament respectively.

\section{Discussion}

The STP is a proposed target for ligament tension as this theoretically avoids the high stiffness regime [7]. The surgeon selected joint force was on average below the STP, indicating the surgeons may already be targeting the STP region, and avoiding the high stiffness regime. It should be noted that Heesterbeek et al. 2017 reported a lower range for the STP, between $48 \mathrm{~N}$ and $59 \mathrm{~N}$ [2].

The medial compartment behaved similarly in extension and flexion. The lateral compartment showed a reduction in stiffness from extension to flexion. For all loading conditions Slope 1 had lower standard deviations during down cycle loading compared to up cycle. Conversely, for all loading conditions Slope 2 had lower standard deviations during up cycle loading compared to down cycle.

The calculated STP had reduced variability when using down cycle data compared to the up cycle data. This was expected due to ligament creep. Future BalanceBot ${ }^{\text {TM }}$ studies should continue to use down cycle data. The flexion data was also more consistent than extension data. Future work is needed to improve extension data collection techniques.

One limitation is the use of a bilinear fit to determine the STP, ignoring the non-linear regime of ligament. Also, the stiffness profiles in some cases were not ideal, showing considerable variability. This could be due to anatomic anomalies, surgeon experience, as well as surgeon technique.

It remains unknown whether the STP is an optimal target. High variability was observed in 
the STP from patient to patient. However, the differentials between the STP and the final joint force were consistent. This result shows that quantifying the patient specific soft tissue profile can assist in determining a target load for the joint space. This work is novel as it is the first study to use intraoperative soft tissue data in extension and flexion to help guide prosthesis implantation.

Future work will investigate how joint tension relative to the STP affects patient outcomes. This will provide insight into optimizing joint tension during TKA.

\section{References}

[1] Hiroshi Asano, Takeshi Muneta, and Akiho Hoshino. Stiffness of soft tissue complex in total knee arthroplasty. Knee Surgery, Sports Traumatology, Arthroscopy, 16(1):51-55, 2008.

[2] PJC Heesterbeek, N Haffner, AB Wymenga, J Stifter, and P Ritschl. Patient-related factors influence stiffness of the soft tissue complex during intraoperative gap balancing in cruciate-retaining total knee arthroplasty. Knee Surgery, Sports Traumatology, Arthroscopy, 25(9):2760-2768, 2017.

[3] Florian Völlner, Tim Weber, Markus Weber, Tobias Renkawitz, Sebastian Dendorfer, Joachim Grifka, and Benjamin Craiovan. A simple method for determining ligament stiffness during total knee arthroplasty in vivo. Scientific reports, 9(1):5261, 2019.

[4] Hendrik Delport, Luc Labey, Ronny De Corte, Bernardo Innocenti, Jos Vander Sloten, and Johan Bellemans. Collateral ligament strains during knee joint laxity evaluation before and after tka. Clinical biomechanics, 28(7):777-782, 2013.

[5] Jeffrey A Weiss, Bradley N Maker, and Sanjay Govindjee. Finite element implementation of incompressible, transversely isotropic hyperelasticity. Computer methods in applied mechanics and engineering, 135(1-2):107-128, 1996.

[6] Stefan Schleifenbaum, Torsten Prietzel, Carsten Hädrich, Robert Möbius, Freddy Sichting, and Niels Hammer. Tensile properties of the hip joint ligaments are largely variable and age-dependent-an in-vitro analysis in an age range of 14-93 years. Journal of biomechanics, 49(14):3437-3443, 2016.

[7] Machacek F Ritschl P, Fuiko R. Evolution in ligament balancing surgical technique for tkr. from historical gap measurement via today's force controlled gap balancing to tomorrows patient's individual joint stability. Conference Proceedings: Computer Assisted Orthopaedic Surgery, page 383-385, 2005. 


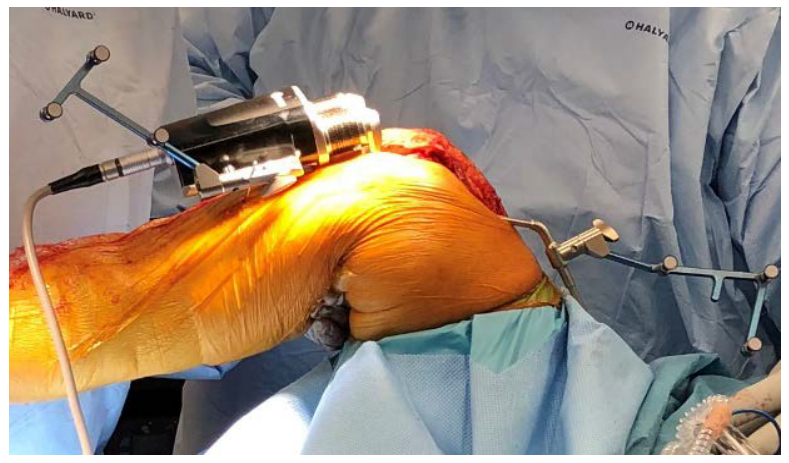

(a) BalanceBot ${ }^{\mathrm{TM}}$ system.
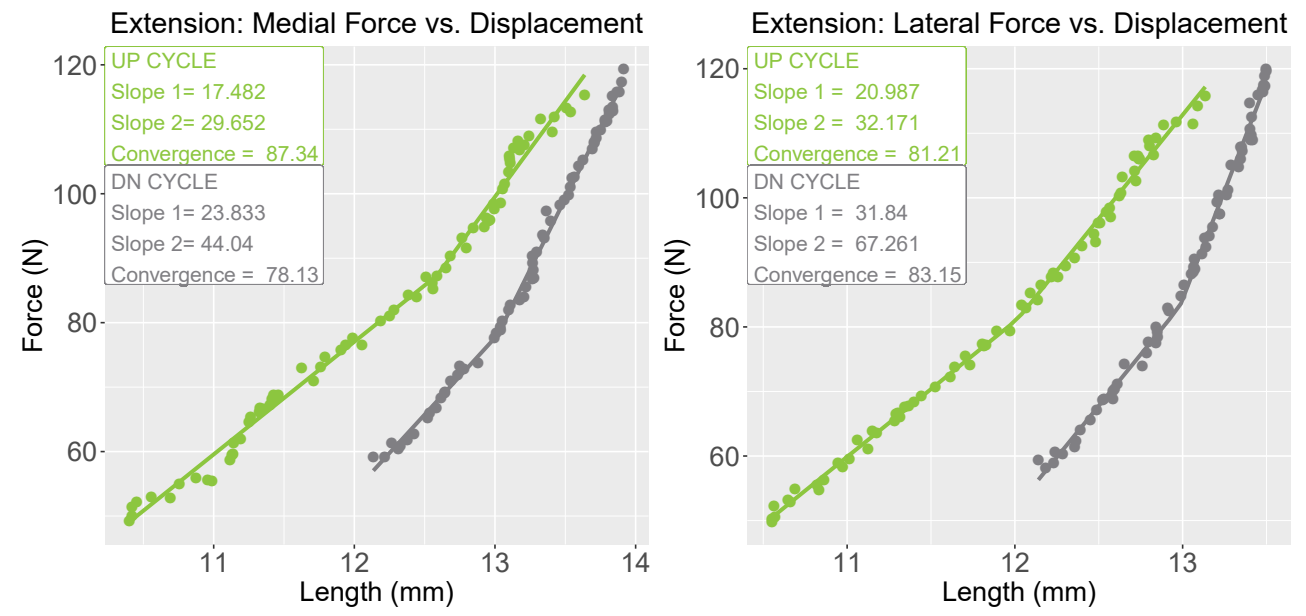

Flexion: Medial Force vs. Displacement

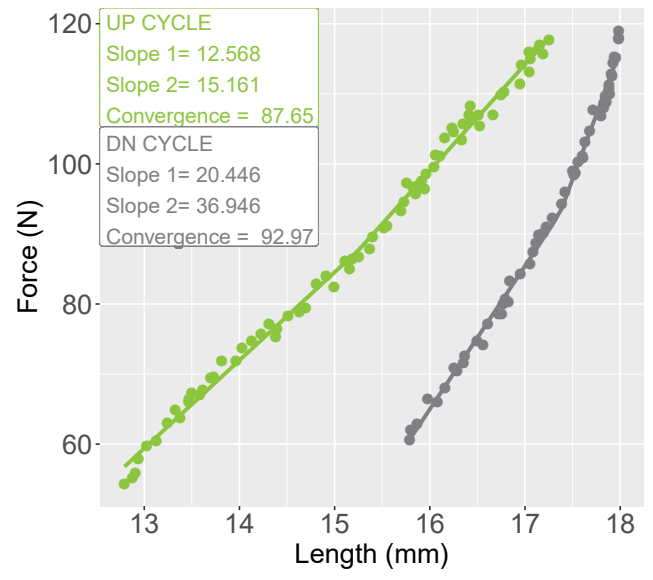

Flexion: Lateral Force vs. Displacement

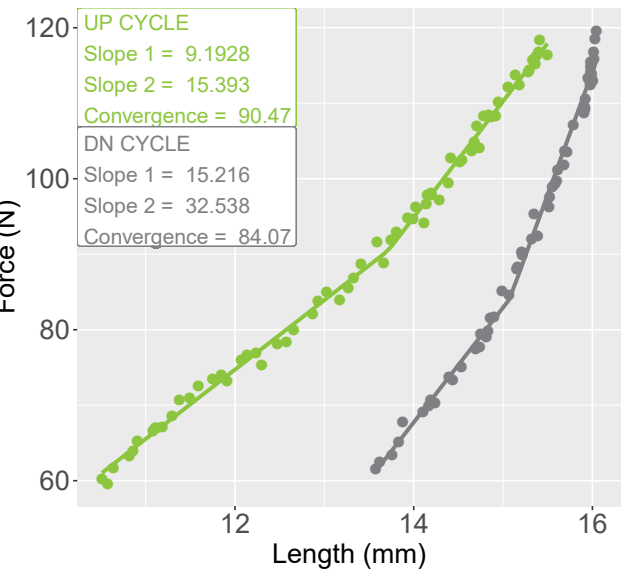

(b) Stiffness profiles from an example patient.

Figure 1: BalanceBot ${ }^{\mathrm{TM}}$ system and example stiffness profiles. The gap in data between the up and down cycles is due to ligament creep. This occurs due to the time taken to switch between the cycles. 